\title{
Static Output-Feedback Control for Vehicle Suspensions: A Single-Step Linear Matrix Inequality Approach
}

\author{
Josep Rubió-Massegú, ${ }^{1}$ Francisco Palacios-Quiñonero, ${ }^{1}$ \\ Josep M. Rossell, ${ }^{1}$ and Hamid Reza Karimi ${ }^{2}$ \\ ${ }^{1}$ Department of Applied Mathematics III, Universitat Politècnica de Catalunya (UPC), Avenue Bases de Manresa 61-73, \\ Manresa 08242, Barcelona, Spain \\ ${ }^{2}$ Department of Engineering, Faculty of Engineering and Science, University of Agder (UiA), 4898 Grimstad, Norway
}

Correspondence should be addressed to Josep Rubió-Massegú; josep.rubio@upc.edu

Received 28 September 2013; Accepted 31 October 2013

Academic Editor: Hui Zhang

Copyright (C) 2013 Josep Rubió-Massegú et al. This is an open access article distributed under the Creative Commons Attribution License, which permits unrestricted use, distribution, and reproduction in any medium, provided the original work is properly cited.

In this paper, a new strategy to design static output-feedback controllers for a class of vehicle suspension systems is presented. A theoretical background on recent advances in output-feedback control is first provided, which makes possible an effective synthesis of static output-feedback controllers by solving a single linear matrix inequality optimization problem. Next, a simplified model of a quarter-car suspension system is proposed, taking the ride comfort, suspension stroke, road holding ability, and control effort as the main performance criteria in the vehicle suspension design. The new approach is then used to design a static output-feedback $H_{\infty}$ controller that only uses the suspension deflection and the sprung mass velocity as feedback information. Numerical simulations indicate that, despite the restricted feedback information, this static output-feedback $H_{\infty}$ controller exhibits an excellent behavior in terms of both frequency and time responses, when compared with the corresponding state-feedback $H_{\mathrm{\infty}}$ controller.

\section{Introduction}

In recent decades, vehicle suspension systems have been attracting a growing interest. In particular, much research effort has been devoted to designing different kinds of passive, active, and semiactive vehicle suspensions using a wide variety of control strategies. Some relevant instances of control strategies used in this field are fuzzy control, optimal control, $H_{\infty}$ control, gain scheduling, adaptive control, and model predictive control [1-5]. The development of these control strategies has been closely related to the emergence of computational tools and efficient numerical algorithms, which allow solving complex and sophisticated control problems in a reasonably short time.

When designing a feedback control system, the amount of information available for feedback purposes is an element of particular importance. In the ideal case that the entire state vector is available, many advanced state-feedback controller designs can be formulated as linear matrix inequality (LMI) optimization problems and efficiently computed using standard computational tools as those provided by the MATLAB Robust Control Toolbox [6]. In a more realistic situation, however, the complete state vector is rarely accessible and the available feedback information consists only in a reduced set of linear combinations of the states. In this context, static output-feedback control strategies are an excellent option to facilitate a simple implementation in practice.

From a computational perspective, static output-feedback controller designs lead to challenging problems. Typically, this kind of problems has been solved using multistep numerical algorithms such as those based on random search [7], or those consisting in iterative procedures [8-11]. In both cases, complex matrix equations or LMI optimization problems need to be solved at each step. To avoid the high computational cost associated with the multistep methods, some single-step strategies have also been proposed [1215], which formulate the static output-feedback controller design in terms of a single LMI optimization problem. These 
single-step methods are based on a proper transformation of the state variables and present the drawback of being highly problem-dependent, in the sense that a complete derivation of the LMI optimization problem must be carried out for most controller designs.

Recently, a new single-step strategy has been presented in [16], which can be applied to any control problem that admits an LMI-based state-feedback controller design. In this case, an LMI formulation to compute the output-feedback control gain matrix can be easily derived by means of a simple change of variables. The new design methodology is computationally effective, conceptually simple, and easy to implement. Moreover, it can be an excellent tool to design static output-feedback controllers in a wide variety of problems by taking advantage of the rich literature on LMI formulations for state-feedback controller design. Some preliminary works, with successful applications to the field of vibration control of large structures, can be found in [17-21].

The main objective of this paper is to explore the potential applicability of the new design methodology in the field of vehicle suspensions. Additionally, we are also interested in providing a clear and practical presentation of the main theoretical elements of the new approach, which we believe can be of general interest for control engineers in different fields. To this end, a static output-feedback $H_{\infty}$ controller is designed for a simplified quarter-car suspension system. A state-feedback $H_{\infty}$ controller is also designed, and it is used as a reference in the performance assessment. Moreover, the LMI formulation of the state-feedback design serves as a natural starting point to derive the LMI formulation for the output-feedback design.

The paper is organized as follows. In Section 2, the fundamental elements of the new strategy for static outputfeedback controller design are summarily discussed. In Section 3, a suitable mathematical model for a quarter-car suspension system is provided, and the general ideas of Section 2 are applied to the particular case of $H_{\infty}$ controller design. In Section 4, a static output-feedback $H_{\infty}$ controller is designed for a particular quarter-car suspension system, and a suitable set of frequency and time responses are computed to assess the effectiveness of the proposed controller. Finally, in Section 5, some conclusions and future lines of research are briefly presented.

\section{Theoretical Background}

Let us consider a linear matrix inequality that depends on a symmetric positive-definite matrix $X \in \mathbb{R}^{n \times n}$, a matrix $Y \in$ $\mathbb{R}^{m \times n}$, and possibly other scalar or matrix variables. Such an LMI can be written in the form:

$$
X>0, \quad F(X, Y, \zeta)>0,
$$

where the vector $\zeta \in \mathbb{R}^{p \times 1}$ collects the free entries of the matrices distinct from $X$ and $Y$, together with the remaining LMI variables, and $F$ is a given affine map that makes the matrix inequality an LMI. This kind of LMI formulation is very common in practice and appears in a large number of state-feedback control problems [22]; some recent works can be found in [23-28]. More precisely, the LMI formulation (1) arises naturally in a wide variety of state-feedback controller designs, where the state control gain matrix $G \in \mathbb{R}^{m \times n}$ is explicitly given by

$$
G=Y X^{-1}
$$

where $X$ is usually the inverse of a Lyapunov matrix or a scaling of it, and $Y$ comes from a previous change of variables defined by

$$
Y=G X
$$

Static output-feedback control problems can be seen as static state-feedback problems with the additional constraint that the state control gain matrix $G$ admits a factorization of the form:

$$
G=K C_{y}
$$

where $K \in \mathbb{R}^{m \times q}$ is the output control gain matrix and $C_{y} \in$ $\mathbb{R}^{q \times n}$ is the observed-output matrix, which is assumed to be a given full row-rank matrix with $q<n$. Consequently, if a static state-feedback control problem can be formulated in terms of an LMI of the form (1) with the state gain matrix given in (2), then the corresponding static output-feedback version of the same control problem can be reduced to a nonconvex problem, consisting in finding matrices $X, Y$, and $\zeta$ satisfying

$$
X>0, \quad F(X, Y, \zeta)>0, \quad(X, Y) \in \mathscr{M},
$$

where $\mathscr{U}$ is the set of all pairs of matrices $(X, Y)$ for which there exists an $m \times q$ matrix $K$ satisfying

$$
Y X^{-1}=K C_{y} \text {. }
$$

Recently, a systematic and easy-to-implement strategy to obtain feasible solutions of (5) has been proposed in [16]. This strategy considers an $n \times(n-q)$ matrix $Q$, whose columns are a basis of $\operatorname{ker}\left(C_{y}\right)$, and a matrix $R$ defined by

$$
R=C_{y}^{\dagger}+Q L
$$

where $L$ is a given $(n-q) \times q$ matrix and

$$
C_{y}^{\dagger}=C_{y}^{T}\left(C_{y} C_{y}^{T}\right)^{-1}
$$

is the Moore-Penrose pseudoinverse of $C_{y}$. Next, the following linear transformations are introduced:

$$
X=Q X_{Q} Q^{T}+R X_{R} R^{T}, \quad Y=Y_{R} R^{T},
$$

where $X_{Q} \in \mathbb{R}^{(n-q) \times(n-q)}$ and $X_{R} \in \mathbb{R}^{q \times q}$ are symmetric matrices and $Y_{R} \in \mathbb{R}^{m \times q}$ is an arbitrary matrix. The design of static output-feedback controllers is based on the following result. 
Theorem 1 (see [16]). If the matrix $X$ in (9) is nonsingular, then $X_{R}$ is also nonsingular and the matrix equation $Y X^{-1}=$ $\mathrm{KC}_{y}$ holds with

$$
K=Y_{R} X_{R}^{-1} .
$$

Moreover, $X$ is positive-definite if and only if the matrices $X_{Q}$ and $X_{R}$ are both positive-definite.

This theorem provides a systematic methodology to obtain solutions of (5). Indeed, we only need to choose a suitable $(n-q) \times q$ matrix $L$ in order to define $R=C_{y}^{\dagger}+Q L$, and solve the following LMI with variables $X_{Q}, X_{R}, Y_{R}$, and $\zeta$ :

$$
\begin{aligned}
& X_{\mathrm{Q}}>0, \quad X_{R}>0, \\
& F\left(Q X_{Q} Q^{T}+R X_{R} R^{T}, Y_{R} R^{T}, \zeta\right)>0,
\end{aligned}
$$

which has been obtained from (1) by using the transformations (9), using also the fact that the condition $X>0$ can be replaced by $X_{Q}>0$ and $X_{R}>0$. If a feasible solution to the LMI (11) is achieved by the matrices $\widetilde{X}_{Q}, \widetilde{X}_{R}, \widetilde{Y}_{R}$, and $\widetilde{\zeta}$ then, for the corresponding matrices $\widetilde{X}, \widetilde{Y}$ defined in (9) and the vector $\widetilde{\zeta}$ we obtain a feasible solution of (5) that, at the same time, satisfies the constraint (6) with the output gain matrix:

$$
K=\widetilde{Y}_{R} \widetilde{X}_{R}^{-1} .
$$

The main features of this strategy are its generality, conceptual simplicity, and ease of implementation. Moreover, it can also be applied to optimization problems of the form:

minimize $h(X, Y, \zeta)$

subject to $X>0, \quad F(X, Y, \zeta)>0, \quad(X, Y) \in \mathscr{M}$,

where the objective function $h$ is assumed to be linear. This kind of optimization problem arises when some performance criterion needs to be optimized. In this case, the optimization problem (13) can be transformed into the following LMI optimization problem with variables $X_{Q}, X_{R}, Y_{R}$, and $\zeta$ :

$$
\begin{array}{ll}
\operatorname{minimize} & \widehat{h}\left(X_{\mathrm{Q}}, X_{R}, Y_{R}, \zeta\right) \\
\text { subject to } & X_{\mathrm{Q}}>0, \quad X_{R}>0, \\
& F\left(Q X_{Q} Q^{T}+R X_{R} R^{T}, Y_{R} R^{T}, \zeta\right)>0,
\end{array}
$$

where the new objective function $\widehat{h}$ is defined as

$$
\widehat{h}\left(X_{\mathrm{Q}}, X_{R}, Y_{R}, \zeta\right)=h\left(\mathrm{QX}_{\mathrm{Q}} \mathrm{Q}^{T}+R X_{R} R^{T}, Y_{R} R^{T}, \zeta\right) .
$$

Clearly, given an optimal solution to the optimization problem (14), a corresponding triplet $(\widetilde{X}, \widetilde{Y}, \widetilde{\zeta})$ of matrices can be computed, which minimizes the objective function $h(X, Y, \zeta)$ on a set that satisfies all the constraints in (13). In particular, we will have $\tilde{Y} \widetilde{X}^{-1}=K C_{y}$ with $K=\widetilde{Y}_{R} \widetilde{X}_{R}^{-1}$.

Remark 2. In general, the objective function $h$ in (13) depends on the variables $X, Y$, and $\zeta$. However, an objective function $h(\zeta)$ that only depends on $\zeta$ will be

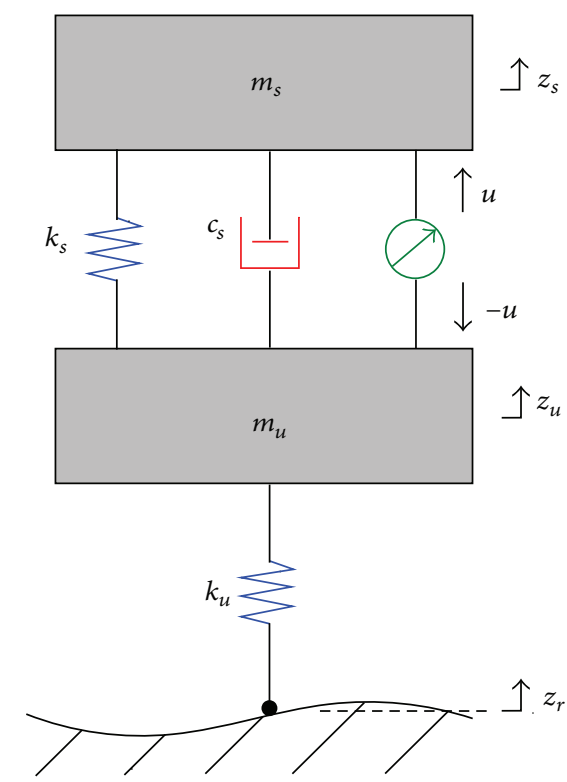

FIGURE 1: Quarter-car suspension model with active suspension.

encountered in the following sections. In this case, we have $\widehat{h}\left(X_{\mathrm{Q}}, X_{R}, Y_{R}, \zeta\right)=h(\zeta)$ and the corresponding LMI optimization problem (14) takes the simplified form:

$$
\begin{array}{cl}
\text { minimize } & h(\zeta) \\
\text { subject to } & X_{\mathrm{Q}}>0, \quad X_{R}>0, \\
& F\left(Q X_{Q} Q^{T}+R X_{R} R^{T}, Y_{R} R^{T}, \zeta\right)>0 .
\end{array}
$$

Remark 3. For simplicity, positive semidefinite terms are not considered in the LMI (1). If necessary, this kind of terms can be easily included by adding a new matrix inequality of the form $F_{0}(X, Y, \zeta) \geq 0$.

\section{Static Output-Feedback Control for Vehicle Suspensions}

In this section, the general methodology introduced in Section 2 is applied to design an output-feedback $H_{\infty}$ control system for a quarter-car suspension model. More precisely, in Section 3.1, a first-order state-space model for a quartercar together with a suitable vector of controlled outputs are presented. An LMI formulation to compute static outputfeedback $H_{\infty}$ controllers is provided in Section 3.2.

3.1. Mathematical Model. Let us consider the quarter-car suspension model schematically depicted in Figure 1. The motion equations can be written as

$$
\begin{aligned}
m_{s} \ddot{z}_{s}(t)= & -c_{s}\left[\dot{z}_{s}(t)-\dot{z}_{u}(t)\right]-k_{s}\left[z_{s}(t)-z_{u}(t)\right] \\
& +u(t), \\
m_{u} \ddot{z}_{u}(t)= & c_{s}\left[\dot{z}_{s}(t)-\dot{z}_{u}(t)\right]+k_{s}\left[z_{s}(t)-z_{u}(t)\right] \\
& -k_{u}\left[z_{u}(t)-z_{r}(t)\right]-u(t),
\end{aligned}
$$


where $m_{s}$ and $m_{u}$ are the sprung and unsprung masses representing the chassis mass and wheel mass, respectively; $k_{s}$ and $c_{s}$ are, respectively, the stiffness and damping of the suspension system; $k_{u}$ stands for the tire stiffness; $z_{r}(t)$ represents the vertical road displacement; $z_{s}(t)$ and $z_{u}(t)$ are the vertical displacements of the sprung and unsprung masses, respectively; and $u(t)$ is the active input of the suspension system. By defining the state variables

$$
\begin{array}{ll}
x_{1}(t)=z_{s}(t), & x_{2}(t)=z_{u}(t), \\
x_{3}(t)=\dot{z}_{s}(t), & x_{4}(t)=\dot{z}_{u}(t),
\end{array}
$$

a first-order state-space model in the form:

$$
\dot{x}(t)=A x(t)+B u(t)+B_{w} w(t)
$$

can be derived, where $x(t)=\left[x_{1}(t), x_{2}(t), x_{3}(t), x_{4}(t)\right]^{T}$ is the vector of states, $u(t)$ is the control input, $w(t)=z_{r}(t)$ is the road disturbance input, and the matrices $A, B$, and $B_{w}$ are given by

$$
\begin{gathered}
A=\left[\begin{array}{cccc}
0 & 0 & 1 & 0 \\
0 & 0 & 0 & 1 \\
-\frac{k_{s}}{m_{s}} & \frac{k_{s}}{m_{s}} & -\frac{c_{s}}{m_{s}} & \frac{c_{s}}{m_{s}} \\
\frac{k_{s}}{m_{u}} & -\frac{k_{s}+k_{u}}{m_{u}} & \frac{c_{s}}{m_{u}} & -\frac{c_{s}}{m_{u}}
\end{array}\right], \\
B=\left[\begin{array}{c}
0 \\
0 \\
\frac{1}{m_{s}} \\
-\frac{1}{m_{u}}
\end{array}\right], \quad B_{w}=\left[\begin{array}{c}
0 \\
0 \\
0 \\
\frac{k_{u}}{m_{u}}
\end{array}\right] .
\end{gathered}
$$

To define the vector of controlled outputs, we consider the ride comfort, suspension stroke, road holding ability, and the required control effort as main performance criteria in the vehicle suspension design. These criteria can be quantified using the sprung mass acceleration $\ddot{z}_{s}(t)$, the suspension deflection $z_{s}(t)-z_{u}(t)$, the tire deflection $z_{u}(t)-z_{r}(t)$, and the control force $u(t)$, respectively, and should be made as small as possible in order to have good vehicle suspension characteristics $[29,30]$. Therefore, we consider the following vector of controlled outputs:

$$
z(t)=\left[\begin{array}{c}
\ddot{z}_{s}(t) \\
\alpha\left(z_{s}(t)-z_{u}(t)\right) \\
\beta\left(z_{u}(t)-z_{r}(t)\right) \\
\eta u(t)
\end{array}\right],
$$

where $\alpha, \beta$, and $\eta$ are adjustable weights that should manage the tradeoff between the above performance requirements. Using (17) to isolate the sprung mass acceleration, the controlled output $z(t)$ can be written as

$$
z(t)=C x(t)+D u(t)+D_{w} w(t)
$$

with

$$
\begin{aligned}
& C=\left[\begin{array}{cccc}
-\frac{k_{s}}{m_{s}} & \frac{k_{s}}{m_{s}} & -\frac{c_{s}}{m_{s}} & \frac{c_{s}}{m_{s}} \\
\alpha & -\alpha & 0 & 0 \\
0 & \beta & 0 & 0 \\
0 & 0 & 0 & 0
\end{array}\right], \\
& D=\left[\begin{array}{c}
1 \\
m_{s} \\
0 \\
0 \\
\eta
\end{array}\right], \quad D_{w}=\left[\begin{array}{c}
0 \\
0 \\
-\beta \\
0
\end{array}\right] \text {. }
\end{aligned}
$$

3.2. Static Output-Feedback $H_{\infty}$ Controller Design. Now, let us consider the system:

$$
\begin{aligned}
& \dot{x}(t)=A x(t)+B u(t)+B_{w} w(t), \\
& z(t)=C x(t)+D u(t)+D_{w} w(t),
\end{aligned}
$$

where $x(t) \in \mathbb{R}^{n}$ is the state vector, $u(t) \in \mathbb{R}^{m}$ is the control input, $w(t) \in \mathbb{R}^{r}$ is the disturbance input, $z(t) \in \mathbb{R}^{d}$ is the controlled output, and $A, B, B_{w}, C, D$, and $D_{w}$ are constant matrices with appropriate dimensions. Given a state-feedback controller:

$$
u(t)=G x(t)
$$

with state gain matrix $G \in \mathbb{R}^{m \times n}$, the following closed-loop system results:

$$
\begin{aligned}
& \dot{x}(t)=A_{G} x(t)+B_{w} w(t), \\
& z(t)=C_{G} x(t)+D_{w} w(t),
\end{aligned}
$$

where

$$
A_{G}=A+B G, \quad C_{G}=C+D G .
$$

For a given control gain matrix $G$, the $H_{\infty}$-norm of the closed-loop system (26) is given by

$$
\gamma_{G}=\left\|T_{G}\right\|_{\infty}=\sup _{\omega \in \mathbb{R}} \sigma_{\max }\left[T_{G}(j \omega)\right],
$$

where $\sigma_{\max }[\cdot]$ denotes the maximum singular value and $T_{G}$ is the transfer function from the disturbance input to the controlled output:

$$
T_{G}(s)=C_{G}\left(s I-A_{G}\right)^{-1} B_{w}+D_{w} .
$$

In the state-feedback $H_{\infty}$ controller design approach, the objective is to obtain a controller of the form (25), which defines an asymptotically stable matrix $A_{G}$ and, simultaneously, minimizes the corresponding $H_{\infty}$-norm $\gamma_{G}$. According to the Bounded Real Lemma [22], for a given scalar $\gamma>0$, the closed-loop state matrix $A_{G}$ is asymptotically stable and $\gamma_{G}<\gamma$, if and only if there exists a symmetric positive-definite matrix $P \in \mathbb{R}^{n \times n}$ such that the matrix inequality

$$
\left[\begin{array}{ccc}
P A_{G}+A_{G}^{T} P & * & * \\
B_{w}^{T} P & -\gamma I & * \\
C_{G} & D_{w} & -\gamma I
\end{array}\right]<0
$$


holds, where $(*)$ denotes the transposed entry. Pre- and postmultiplying both sides of (30) by the symmetric matrix $\operatorname{diag}[X, I, I]$ with $X=P^{-1}$, using the values of the matrices $A_{G}$, and $C_{G}$ in (27), and introducing the new variable $Y=$ $G X$, we arrive at the linear matrix inequality:

$$
S(X, Y, \gamma)=\left[\begin{array}{ccc}
A X+X A^{T}+B Y+Y^{T} B^{T} & * & * \\
B_{w}^{T} & -\gamma I & * \\
C X+D Y & D_{w} & -\gamma I
\end{array}\right]<0 .
$$

The state-feedback $H_{\infty}$ controller can then be effectively computed by solving the following LMI optimization problem:

$$
\begin{array}{ll}
\text { minimize } & \gamma \\
\text { subject to } & X>0, \quad \gamma>0, \quad S(X, Y, \gamma)<0 .
\end{array}
$$

If the optimization problem (32) attains an optimal value $\tilde{\gamma}$ for the matrices $\widetilde{X}$ and $\widetilde{Y}$, then the state gain matrix $G=$ $\tilde{Y} \widetilde{X}^{-1}$ defines a state-feedback controller $u(t)=G x(t)$ with an asymptotically stable closed-loop state matrix $A_{G}$ and an optimal $H_{\infty}$-norm $\gamma_{G}=\tilde{\gamma}$.

Let us now consider the case of a static output-feedback controller:

$$
u(t)=K y(t)
$$

where $K \in \mathbb{R}^{m \times q}$ is the observed-output gain matrix and $y(t) \in \mathbb{R}^{q}$ is the vector of observed outputs, which can be written as

$$
y(t)=C_{y} x(t)
$$

for a given full row-rank matrix $C_{y} \in \mathbb{R}^{q \times n}$ with $q<n$. From (33) and (34), we obtain

$$
u(t)=K C_{y} x(t)
$$

and consequently, the output-feedback controller (33) can be considered as a state-feedback controller with an associated state gain matrix $G_{\text {of }}=K C_{y}$. Therefore, the design of a static output-feedback $H_{\infty}$ controller leads to the optimization problem (32) with the additional constraint $Y X^{-1}=K C_{y}$, which is a particular case of the optimization problem (13) with

$$
\begin{gathered}
\zeta=\gamma, \quad h(X, Y, \gamma)=\gamma, \\
F(X, Y, \gamma)=\operatorname{diag}[\gamma,-S(X, Y, \gamma)] .
\end{gathered}
$$

According to the discussion presented in Section 2, the following LMI optimization problem results:

$$
\begin{array}{ll}
\operatorname{minimize} & \gamma \\
\text { subject to } & X_{\mathrm{Q}}>0, \quad X_{R}>0, \quad \gamma>0, \\
& S\left(Q X_{\mathrm{Q}} Q^{T}+R X_{R} R^{T}, Y_{R} R^{T}, \gamma\right)<0,
\end{array}
$$

where the matrix inequality $S\left(Q X_{Q} Q^{T}+R X_{R} R^{T}, Y_{R} R^{T}, \gamma\right)<0$ takes the explicit form

$$
\left[\begin{array}{ccc}
A Q X_{\mathrm{Q}} Q^{T}+Q X_{\mathrm{Q}} Q^{T} A^{T}+A R X_{R} R^{T}+R X_{R} R^{T} A^{T}+B Y_{R} R^{T}+R Y_{R}^{T} B^{T} & * & * \\
B_{w}^{T} & -\gamma I & * \\
C Q X_{\mathrm{Q}} Q^{T}+C R X_{R} R^{T}+D Y_{R} R^{T} & D_{w} & -\gamma I
\end{array}\right]<0 .
$$

If the optimization problem defined in (37) attains an optimal solution $\tilde{\gamma}$ for the matrices $\widetilde{X}_{Q}, \widetilde{X}_{R}$, and $\widetilde{Y}_{R}$, then the output gain matrix $K=\widetilde{Y}_{R} \widetilde{X}_{R}^{-1}$ defines a static output-feedback controller $u(t)=K y(t)$ with asymptotically stable matrix $A_{G_{\text {of }}}$ and optimal $H_{\infty}$-norm $\gamma_{G_{\text {of }}} \leq \tilde{\gamma}$, where $G_{\text {of }}=K C_{y}$.

Remark 4. Note that the LMI optimization problem (37) allows us to design a static output-feedback $H_{\infty}$ controller $u(t)=K y(t)$ by solving a single LMI optimization problem. Moreover, the optimal value $\tilde{\gamma}$ provides an upper bound of the $H_{\infty}$-norm corresponding to $G_{\text {of }}=K C_{y}$. The actual value of $\gamma_{G_{\text {of }}}$ can be obtained by maximizing the maximum singular value of the matrix $T_{G_{\mathrm{o}}}(j \omega)$, as indicated in (28). Alternatively, the value $\gamma_{G_{\text {of }}}$ can also be computed by solving the LMI optimization problem:

$$
\begin{array}{ll}
\text { minimize } & \gamma \\
\text { subject to } & P>0, \quad \gamma>0, \quad \text { LMI (30). }
\end{array}
$$

\section{Numerical Results}

In this section, the ideas presented in Section 3.2 are applied to design a static output-feedback $H_{\infty}$ controller for the quarter-car suspension model described in Section 3.1. This output-feedback controller only uses the suspension deflection and the sprung mass velocity as feedback information. A state-feedback $H_{\infty}$ controller is also computed to be taken as a reference in the performance assessment. Next, three different configurations are considered: (i) uncontrolled system, (ii) controlled system using the state-feedback $H_{\infty}$ controller, and (iii) controlled system using the static outputfeedback $H_{\infty}$ controller. For these configurations, a brief discussion on the corresponding frequency responses is presented in Section 4.2. The time responses to an isolated bump as road disturbance are presented and discussed in Section 4.3. Finally, some closing remarks are provided in Section 4.4. All computations have been carried out with 
the MATLAB Robust Control Toolbox [6], and the following particular values $[29,31]$

$$
\begin{gathered}
m_{s}=504.5 \mathrm{~kg}, \quad m_{u}=62 \mathrm{~kg}, \quad k_{s}=13100 \mathrm{~N} / \mathrm{m}, \\
k_{u}=252000 \mathrm{~N} / \mathrm{m}, \quad c_{s}=400 \mathrm{Ns} / \mathrm{m},
\end{gathered}
$$

have been taken as parameters of the quarter-car suspension model in the controllers design and numerical simulations.

4.1. Controllers Design. Let us consider the quarter-car statespace model defined by (19), (20), and the parameter values given in (40). To design a state-feedback $H_{\infty}$ controller

$$
u(t)=G_{\mathrm{sf}} x(t),
$$

which uses the full state vector given in (18) as feedback information, we also consider the controlled output defined in (22), (23) and the following particular values of the weighting coefficients:

$$
\alpha=8, \quad \beta=10, \quad \eta=1.5 \times 10^{-3} .
$$

By solving the LMI optimization problem given in (32), we obtain the state gain matrix:

$$
G_{\text {sf }}=10^{3} \times\left[\begin{array}{llll}
1.4733 & -5.0315 & -2.8818 & 0.1018
\end{array}\right],
$$

with an associated $H_{\infty}$-norm:

$$
\gamma_{G_{\text {sf }}}=528.32 \text {. }
$$

Now, as proposed in [29], let us assume that the available feedback information only includes the suspension deflection and the sprung mass velocity. In this case, the vector of observed outputs is

$$
y(t)=\left[\begin{array}{ll}
z_{s}(t)-z_{u}(t) & \dot{z}_{s}(t)
\end{array}\right]^{T},
$$

which can be written in the form:

$$
y(t)=C_{y} x(t)
$$

where $x(t)$ is the state vector defined in (18), and

$$
C_{y}=\left[\begin{array}{cccc}
1 & -1 & 0 & 0 \\
0 & 0 & 1 & 0
\end{array}\right]
$$

is the observed-output matrix. To design a static outputfeedback $H_{\infty}$ controller

$$
u(t)=K y(t),
$$

we compute the matrix

$$
Q=\left[\begin{array}{ll}
1 & 0 \\
1 & 0 \\
0 & 0 \\
0 & 1
\end{array}\right],
$$

whose columns are a basis of $\operatorname{ker}\left(C_{y}\right)$ and the matrix

$$
R=\left[\begin{array}{cc}
\frac{1}{2} & 0 \\
-\frac{1}{2} & 0 \\
0 & 1 \\
0 & 0
\end{array}\right],
$$

which can be obtained from (7) for the particular choice $L=0$. By solving the LMI optimization problem (37) with the matrices $Q$ and $R$ given in (49), (50) and the same matrices $A, B, B_{w}, C, D$, and $D_{w}$ used in the previous state-feedback controller design, we get the observed-output gain matrix:

$$
K=10^{3} \times\left[\begin{array}{ll}
-0.3585 & -8.9459
\end{array}\right],
$$

and an optimal $\gamma$-value:

$$
\widetilde{\gamma}=551.03 .
$$

According to Remark 4 and the $\gamma$-value in (44), the $H_{\infty}$ norm of the state gain matrix

$$
G_{\text {of }}=K C_{y}
$$

associated with the observed-output gain matrix $K$, satisfies

$$
528.32 \leq \gamma_{G_{0 f}} \leq 551.03 .
$$

By setting $G=G_{\text {of }}$ in (30) and solving the optimization problem (39), the following value of $\gamma_{G_{\text {of }}}$ results:

$$
\gamma_{G_{\text {of }}}=532.74,
$$

which is only a $0.84 \%$ greater than the optimal value $\gamma_{G_{s f}}$ achieved by the state-feedback $H_{\infty}$ controller.

4.2. Frequency Response. In this subsection, we consider the frequency response for three different control configurations of the quarter-car suspension model: (i) uncontrolled system, (ii) controlled system using the state-feedback $H_{\infty}$ controller defined in (41) and (43), and (iii) controlled system using the static output-feedback $H_{\infty}$ controller defined in (45), (48), and (51). For these configurations, the frequency transfer functions from the road displacement $w(t)$ to the magnitudes used as performance criteria are displayed in Figure 2. Specifically, the frequency transfer functions from the road displacement $w(t)$ to the sprung mass acceleration $\ddot{z}_{s}(t)$ are presented in Figure 2(a), where the black dotted line corresponds to the uncontrolled system (denoted as Passive in the legend), the blue dashed line pertains to the state-feedback controller (denoted as State-feedback in the legend), and the red solid line corresponds to the static output-feedback controller (denoted as Output-feedback in the legend). The frequency transfer functions from the road displacement $w(t)$ to the suspension deflection $z_{s}(t)-z_{u}(t)$, tire deflection $z_{u}(t)-z_{r}(t)$, and control force $u(t)$ are displayed in Figures 2(b), 2(c), and 2(d), respectively, using the same line styles and colors. 


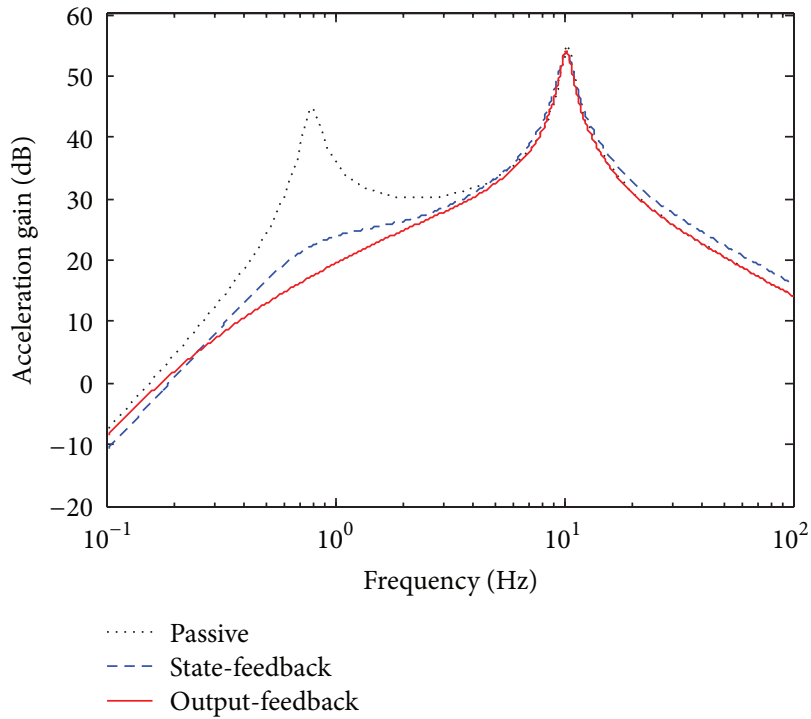

(a)

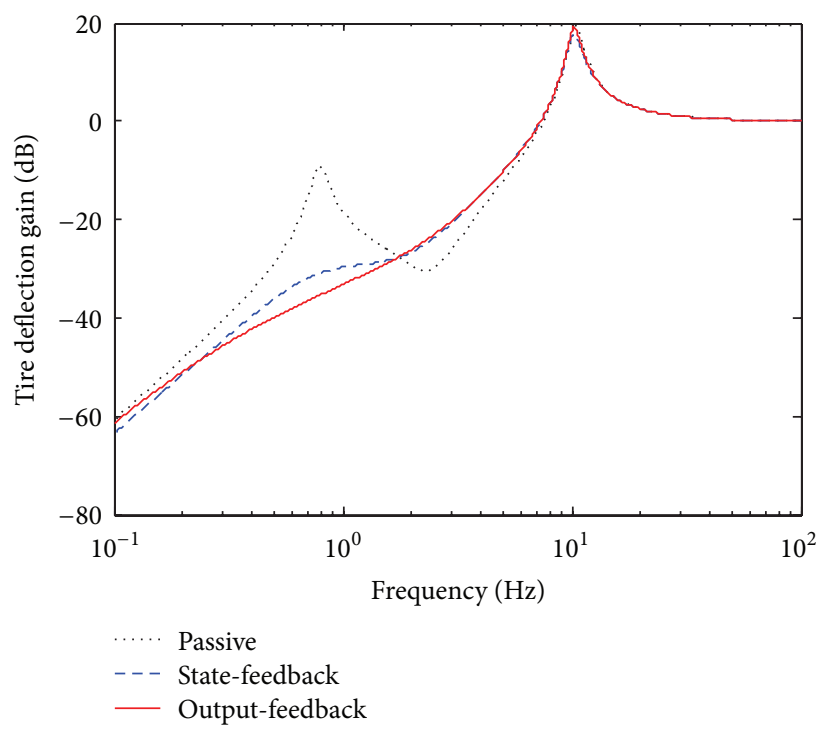

(c)

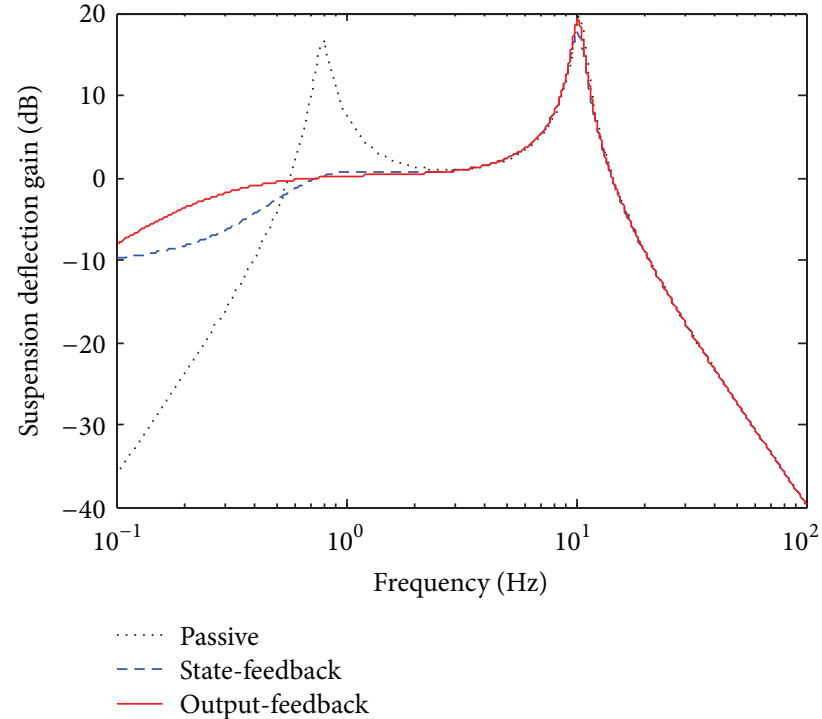

(b)

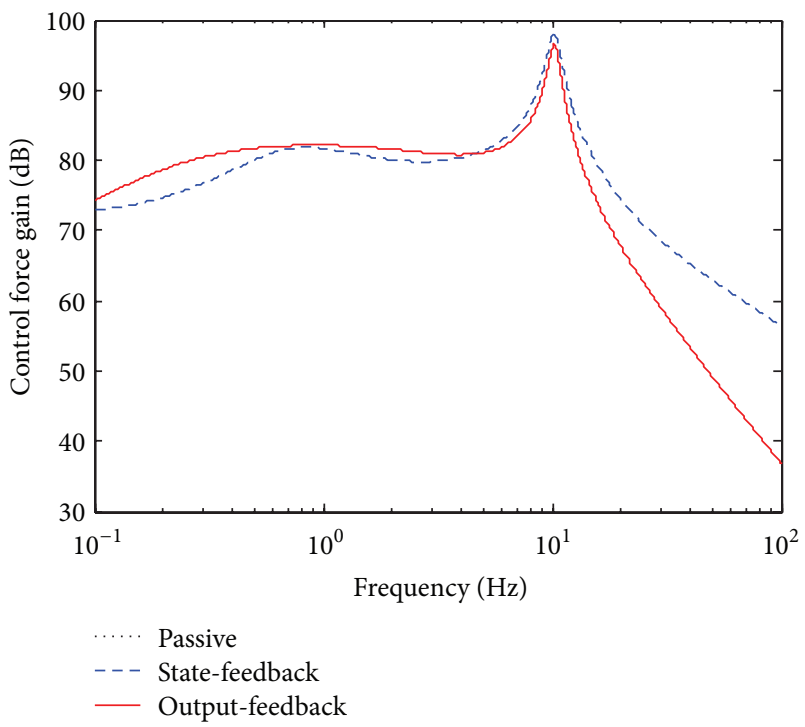

(d)

FIGURE 2: Frequency transfer functions from road displacement to (a) sprung mass acceleration, (b) suspension deflection, (c) tire deflection, and (d) control effort, corresponding to the passive (black dotted line), state-feedback (blue dashed line), and static output-feedback (red solid line) configurations.

Looking at the graphics in Figure 2(a), it can be appreciated that a significant improvement on ride comfort is provided by the state-feedback and output-feedback controllers when compared with the passive system, especially in the sensitive frequency range of $0-65 \mathrm{rad} / \mathrm{s}$ [32]. A closer look at the graphics corresponding to the active control configurations in Figures 2(a) and 2(d) also indicates that, in this case, the static output-feedback controller behaves slightly better than the state-feedback controller requiring, moreover, similar levels of control effort. Regarding the suspension deflection and tire deflection, the graphics in Figures 2(b) and 2(c) show that both active controllers provide a significant improvement near the natural frequency of the sprung mass mode:

$$
f_{s}=\sqrt{\frac{k_{s}}{m_{s}}},
$$

which for our particular model takes the value $f_{s}=5.1 \mathrm{rad} / \mathrm{s}$ $(0.81 \mathrm{~Hz})$. It should be highlighted that, in the case of suspension deflection (Figure 2(b)), the passive control configuration exhibits the best behavior for frequencies inferior to $0.6 \mathrm{~Hz}$. However, it should also be noted that in this case both active control configurations present negative $\mathrm{dB}$-gains for frequencies below $0.6 \mathrm{~Hz}$. 
Remark 5. As indicated in [33], independently of the suspension type (passive or active), the frequency transfer function from road displacement to sprung mass acceleration has an invariant point at the wheel-hop frequency:

$$
f_{u}=\sqrt{\frac{k_{u}}{m_{u}}}
$$

with magnitude $k_{u} / m_{s}$. This fact reveals the difficulty of improving the ride comfort for frequencies around $f_{u}$. For the parameter values in (40), we have a wheel-hop frequency of $f_{u}=63.75 \mathrm{rad} / \mathrm{s}(10.15 \mathrm{~Hz})$ with a magnitude of $k_{u} / m_{s}=$ $499.50(53.97 \mathrm{~dB})$. The existence of an invariant point also applies to the frequency transfer function from road displacement to suspension deflection at the rattle-space frequency:

$$
f_{r}=\sqrt{\frac{k_{u}}{m_{s}+m_{u}}}
$$

which for the parameters in (40) takes the value $f_{r}=$ $21.10 \mathrm{rad} / \mathrm{s}(3.36 \mathrm{~Hz})$.

Remark 6. The graphics of frequency response in Figure 2 use the road displacement as disturbance input. For the sake of completeness, the graphics of frequency response using the road displacement velocity as disturbance input are presented in Figure 3. Note that the comments made to the graphics in Figure 2 also apply to this second set of graphics.

4.3. Time Response to a Bump Disturbance. To provide a more complete picture of the performance achieved by the proposed static output-feedback controller, in this subsection we present the time response of the quarter-car suspension system to a road disturbance. More precisely, we consider an isolated bump of the form:

$$
z_{r}(t)= \begin{cases}\frac{H}{2}\left[1-\cos \left(\frac{2 \pi V}{L} t\right)\right] & \text { if } 0 \leq t \leq \frac{L}{V} \\ 0 & \text { otherwise }\end{cases}
$$

where $H$ and $L$ are the bump height and length, respectively; and $V$ is the vehicle velocity. The following particular values of the parameters [30]:

$$
H=0.1 \mathrm{~m}, \quad L=5 \mathrm{~m}, \quad V=12.5 \mathrm{~m} / \mathrm{s},
$$

have been used to conduct the numerical simulations. For this road disturbance, the magnitudes taken as performance criteria have been computed for the control configurations (i)-(iii) defined in Section 4.2, and the corresponding graphics are presented in Figure 4. In particular, the graphics in Figure 4(a) display the sprung mass acceleration $\ddot{z}_{s}(t)$ for the uncontrolled system (black dotted line), the controlled system with state-feedback controller (blue dashed line), and the controlled system with static output-feedback controller (red solid line). The graphics of suspension deflection $z_{s}(t)-z_{u}(t)$, tire deflection $z_{u}(t)-z_{r}(t)$, and control force $u(t)$ for these three control configurations are displayed in Figures 4(b), 4(c), and 4(d), respectively, using the same line styles and colors.

The graphics in Figures 4(a)-4(c) clearly show that a significant improvement in ride comfort, suspension deflection, and road holding ability is provided by the active controllers. It can also be appreciated that the static output-feedback controller achieves practically the same levels of vibrational response mitigation as the state-feedback controller. Moreover, the graphics in Figure 4(d) point out that there are no relevant differences between the levels of control effort required to operate the active controllers.

4.4. Closing Remarks. The numerical results obtained in this section indicate that the proposed static output-feedback $H_{\infty}$ controller exhibits a remarkable performance in terms of both frequency and time responses when compared with the corresponding state-feedback $H_{\infty}$ controller. In fact, from the point of view of $H_{\infty}$ controller design, the values of the $H_{\infty^{-}}$ norms in (44) and (55) show that the static output-feedback controller is practically optimal.

These outstanding numerical results are even more meaningful when considering some additional features of the new design methodology: (i) Generality: the proposed methodology can be applied to a wide variety of control problems, with the only requirement that the state-feedback version of the problem admits a standard LMI formulation. (ii) Conceptual simplicity: the ideas involved in the proposed change of variables are simple and transparent. As shown in Section 2, new LMI formulations for static output-feedback controller designs can be easily derived from existing statefeedback LMI formulations through a simple change of the LMI variables. (iii) Ease of implementation: the static output-feedback controller design is formulated in terms of LMI optimization problems, which can be directly solved using standard computational tools, as those provided by the MATLAB Robust Control Toolbox [6]. (iv) Computational efficiency: traditionally, static output-feedback gain matrices have been computed by means of multistep optimization algorithms that require solving complex matrix equations or LMI problems at each step. In contrast, in the new design methodology, the output-feedback gain matrix is computed by solving a single LMI optimization problem.

The property of computational efficiency deserves some additional considerations. As it is well known, the nonconvex nature of static output-feedback control problems makes them NP-hard [34, 35]. Consequently, heuristic strategies are commonly used to solve this kind of problems in a computationally effective way. These heuristic strategies can be based on a certain set of sufficient conditions for controller design or on sophisticated randomized algorithms. In any case, the design procedure depends critically on a suitable choice of certain parameters that, in principle, can take a wide range of possible values.

Obviously, the previous remark also applies to the new design strategy proposed in this paper, which requires a proper choice of the matrix $L$ in (7) to define the change of variables (9). The output-feedback controller design presented in Section 4.1 has been carried out by taking $L$ as a zero matrix, which leads to the simplified form $R=C_{y}^{\dagger}$ 


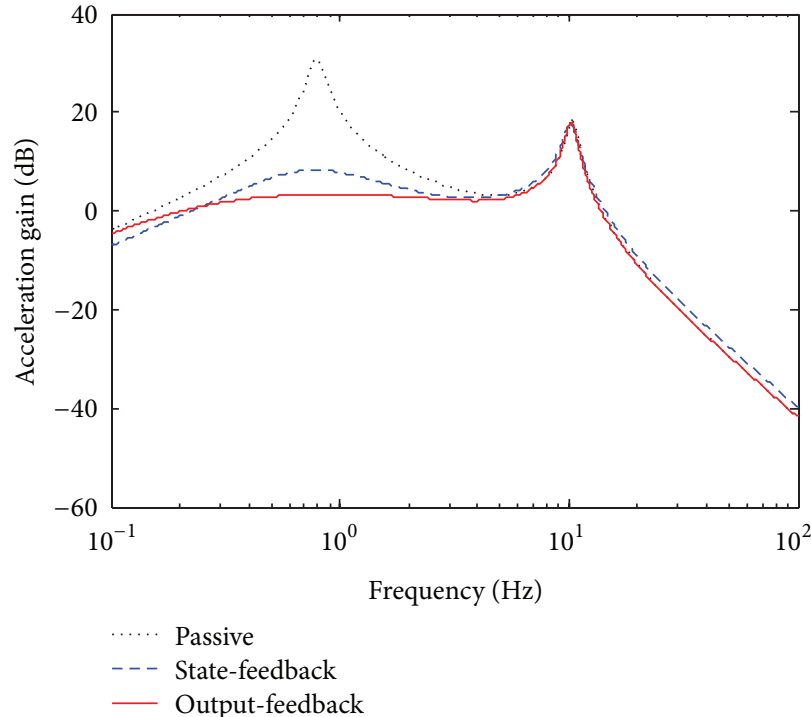

(a)

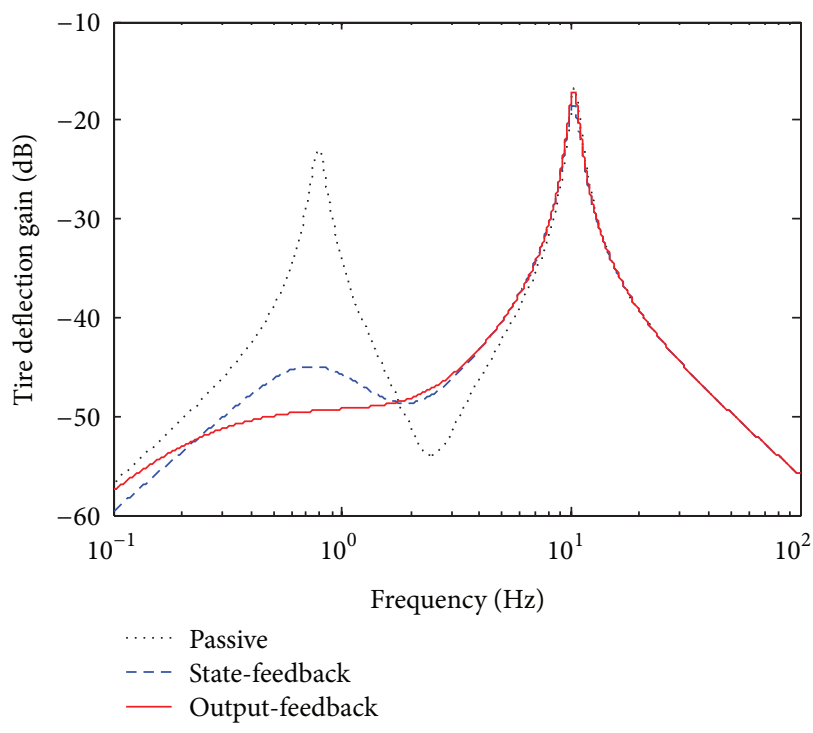

(c)

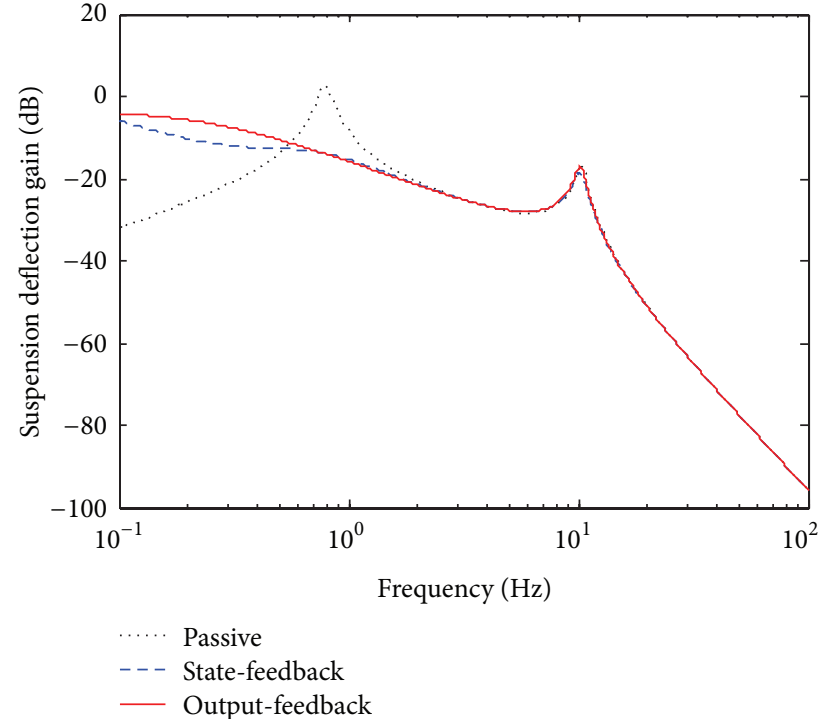

(b)

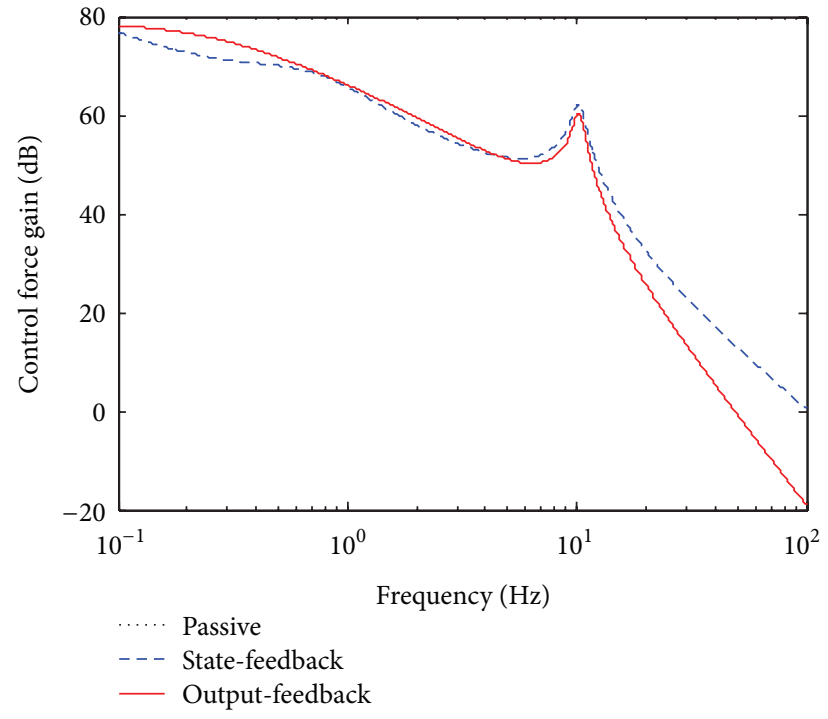

(d)

Figure 3: Frequency transfer functions from road displacement velocity to (a) sprung mass acceleration, (b) suspension deflection, (c) tire deflection, and (d) control effort, corresponding to the passive (black dotted line), state-feedback (blue dashed line), and static output-feedback (red solid line) control configurations.

in (7). The choice $L=0$ has also been used recently in the field of vibration control of large structures with positive results [17-21]. However, it is worth pointing out that certain feasibility problems typically appear when applying the proposed methodology to the design of static outputfeedback controllers for structural vibration control, and that ongoing investigations indicate that a suitable choice of the $L$ matrix can play an important role in solving these feasibility issues.

\section{Conclusions and Future Directions}

In this work, a new strategy to design static output-feedback controllers for vehicle suspension systems has been presented. The proposed strategy is conceptually simple, easy to implement, and computationally efficient, and it can be applied to a wide variety of control problems, with the only requirement that the state-feedback version of the problem admits a standard LMI formulation. To illustrate the main elements of the new approach, a static output-feedback $H_{\infty}$ controller has been designed for a simplified quarter-car suspension system. Numerical simulations show that the proposed static output-feedback $H_{\infty}$ controller exhibits a remarkable behavior in terms of both frequency and time responses, when compared with the corresponding state-feedback $H_{\infty}$ controller.

The main contribution of the paper is twofold: (i) to provide a clear and practical presentation of the main 


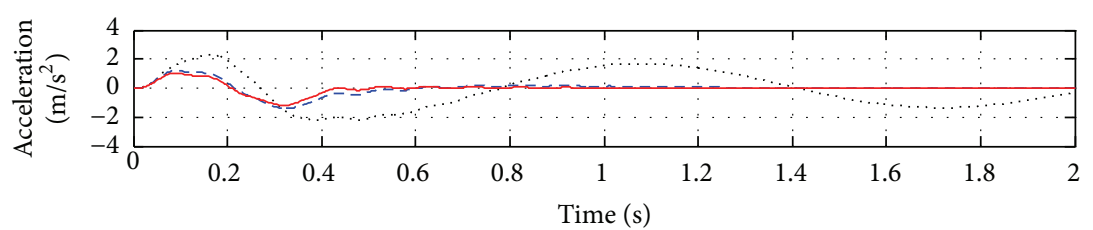

(a)

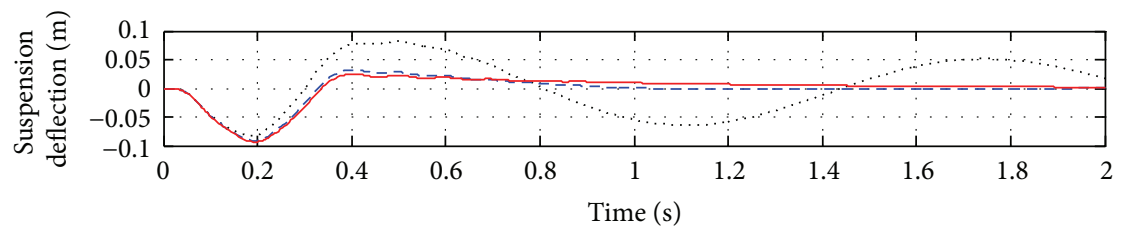

(b)

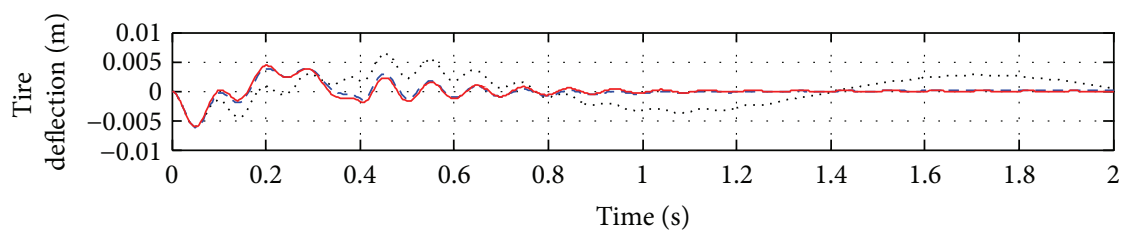

(c)

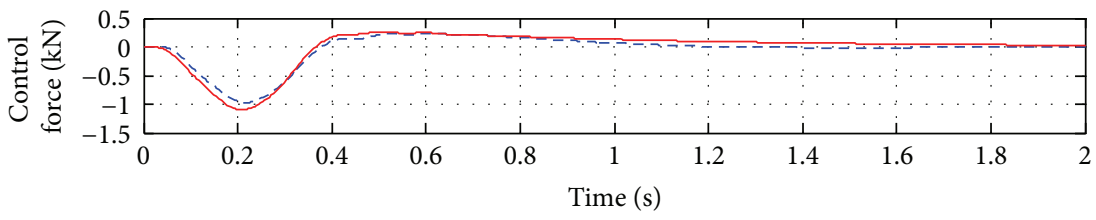

(d)

FiguRE 4: Time response to an isolated bump disturbance: (a) sprung mass acceleration, (b) suspension deflection, (c) tire deflection, and (d) control effort, corresponding to the passive (black dotted line), static state-feedback (blue dashed line), and static output-feedback (red solid line) control configurations.

theoretical elements of the new strategy for static outputfeedback controller design and (ii) to show the practical relevance of the proposed design strategy in the field of automotive suspensions. Indeed, the positive results obtained for the quarter-car suspension system clearly indicate that more research effort should be invested in applying the new design methodology to more complex scenarios. Specifically, more complete physical models can be considered, as half or full car models, seat suspension systems, and humanbody models $[4,36,37]$. More sophisticated control strategies should be also explored, as those including multiobjective designs with $H_{\infty}$ and generalized $H_{2}$ control strategies, fuzzy control, advanced $H_{\infty}$ control, or model predictive control $[2,3,28,36,38-43]$, combined with different mathematical complexities as, for example, uncertainties, input and output constraints, actuation saturations, delays and actuator faults $[23,24,26,30,36,44-46]$, and also combined with actuator dynamics and road excitation models $[39,47]$.

\section{Conflict of Interests}

The authors declare that there is no conflict of interests regarding the publication of this paper.

\section{Acknowledgments}

This work was supported in part by the Spanish Ministry of Economy and Competitiveness under Grant DPI2012$32375 / F E D E R$ and by the Norwegian Center of Offshore Wind Energy (NORCOWE) under Grant 193821/S60 from the Research Council of Norway (RCN). NORCOWE is a consortium with partners from industry and science, hosted by Christian Michelsen Research.

\section{References}

[1] I. Fialho and G. J. Balas, "Road adaptive active suspension design using linear parameter-varying gain-scheduling," IEEE Transactions on Control Systems Technology, vol. 10, no. 1, pp. 43-54, 2002.

[2] H. Chen and C. W. Scherer, "An LMI based model predictive control scheme with guaranteed $H_{\infty}$ performance and its application to active suspension," in Proceedings of the American Control Conference (AAC '04), pp. 1487-1492, Boston, Mass, USA, July 2004.

[3] H. Du and N. Zhang, "Fuzzy control for nonlinear uncertain electrohydraulic active suspensions with input constraint," IEEE Transactions on Fuzzy Systems, vol. 17, no. 2, pp. 343-356, 2009. 
[4] H. Li, H. Gao, and H. Liu, "Robust quantised control for active suspension systems," IET Control Theory \& Applications, vol. 5, no. 17, pp. 1955-1969, 2011.

[5] R. S. Prabakar, C. Sujatha, and S. Narayanan, "Response of a quarter car model with optimal magnetorheological damper parameters," Journal of Sound and Vibration, vol. 332, no. 9, pp. 2191-2206, 2013.

[6] G. Balas, R. Chiang, A. Packard, and M. Safonov, MATLAB Robust Control Toolbox User's Guide, Version 4.2, The MathWorks, Inc., Natick, Mass, USA, 2012.

[7] R. Toscano, "A simple method to find a robust output feedback controller by random search approach," ISA Transactions, vol. 45, no. 1, pp. 35-44, 2006.

[8] D. D. Moerder and A. J. Calise, "Convergence of a numerical algorithm for calculating optimal output feedback gains," IEEE Transactions on Automatic Control, vol. 30, no. 9, pp. 900-903, 1985.

[9] Y.-Y. Cao, J. Lam, and Y.-X. Sun, "Static output feedback stabilization: an ILMI approach," Automatica, vol. 34, no. 12, pp. 1641-1645, 1998.

[10] J. Gadewadikar, F. L. Lewis, L. Xie, V. Kucera, and M. AbuKhalaf, "Parameterization of all stabilizing $H_{\infty}$ static statefeedback gains: application to output-feedback design," Automatica, vol. 43, no. 9, pp. 1597-1604, 2007.

[11] J.-E. Feng, J. Lam, P. Li, and Z. Shu, "Decay rate constrained stabilization of positive systems using static output feedback," International Journal of Robust and Nonlinear Control, vol. 21, no. 1, pp. 44-54, 2011.

[12] C. A. R. Crusius and A. Trofino, "Sufficient LMI conditions for output feedback control problems," IEEE Transactions on Automatic Control, vol. 44, no. 5, pp. 1053-1057, 1999.

[13] E. Prempain and I. Postlethwaite, "Static output feedback stabilisation with $H_{\infty}$ performance for a class of plants," Systems and Control Letters, vol. 43, no. 3, pp. 159-166, 2001.

[14] G. I. Bara and M. Boutayeb, "Static output feedback stabilization with $H_{\infty}$ performance for linear discrete-time systems," IEEE Transactions on Automatic Control, vol. 50, no. 2, pp. 250-254, 2005.

[15] M. Chaabane, F. Tadeo, D. Mehdi, and M. Souissi, "Robust admissibilization of descriptor systems by static outputfeedback: an LMI approach," Mathematical Problems in Engineering, vol. 2011, Article ID 960981, 10 pages, 2011.

[16] J. Rubió-Massegú, J. M. Rossell, H. R. Karimi, and F. PalaciosQuiñonero, "Static output-feedback control under information structure constraints," Automatica, vol. 49, no. 1, pp. 313-316, 2013.

[17] J. Rubió-Massegú, F. Palacios-Quiñonero, and J. M. Rossell, "Decentralized static output-feedback $H_{\infty}$ controller design for buildings under seismic excitation," Earthquake Engineering and Structural Dynamics, vol. 41, no. 7, pp. 1199-1205, 2012.

[18] F. Palacios-Quiñonero, J. Rubió-Massegú, J. M. Rossell, and H. R. Karimi, "Optimal passive-damping design using a decentralized velocity-feedback $H_{\infty}$ approach," Modeling, Identification and Control, vol. 33, no. 3, pp. 87-97, 2012.

[19] F. Palacios-Quiñonero, J. Rubió-Massegú, J. M. Rossell, and H. R. Karimi, "Discrete-time static output-feedback semidecentralized $H_{\infty}$ controller design: an application to structural vibration control," in Proceedings of the American Control Conference, pp. 6126-6131, Montreal, Canada, 2012.

[20] F. Palacios-Quiñonero, H. R. Karimi, J. Rubió-Massegú, and J. M. Rossell, "Passive-damping design for vibration control of large structures," in Proceedings of the 10th IEEE International Conference on Control and Automation, pp. 33-38, Hangzhou, China, 2013.

[21] F. Palacios-Quiñonero, J. Rubió-Massegú, J. M. Rossell, and H. R. Karimi, "Vibration control for adjacent structures using local state information," Mechatronics, 2014.

[22] S. Boyd, L. El Ghaoui, E. Feron, and V. Balakrishnan, Linear Matrix Inequalities in System and Control Theory, vol. 15 of SIAM Studies in Applied Mathematics, SIAM, Philadelphia, Pa, USA, 1994.

[23] X. Yang, H. Gao, P. Shi, and G. Duan, "Robust $H_{\infty}$ control for a class of uncertain mechanical systems," International Journal of Control, vol. 83, no. 7, pp. 1303-1324, 2010.

[24] A. Chen and J. Wang, "Delay-dependent $L_{2}-L_{\infty}$ control of linear systems with multiple time-varying state and input delays," Nonlinear Analysis: Real World Applications, vol. 13, no. 1, pp. 486-496, 2012.

[25] F. Amato, R. Ambrosino, M. Ariola, and A. Merola, "Domain of attraction and guaranteed cost control for non-linear quadratic systems. Part 2: controller design," IET Control Theory \& Applications, vol. 7, no. 4, pp. 565-572, 2013.

[26] Z. Gu, D. Yue, C. Peng, J. Liu, and J. Zhang, "Fault tolerant control for systems with interval time-varying delay and actuator saturation," Journal of the Franklin Institute, vol. 350, no. 2, pp. 231-243, 2013.

[27] P.-L. Liu, "State feedback stabilization of time-varying delay uncertain systems: a delay decomposition approach," Linear Algebra and Its Applications, vol. 438, no. 5, pp. 2188-2209, 2013.

[28] H. Xia, P. Zhao, L. Li, A. Wu, and G. Ma, "A novel approach to $H_{\infty}$ control design for linear neutral time-delay systems," Mathematical Problems in Engineering, vol. 2013, Article ID 526017, 9 pages, 2013.

[29] H. Du, J. Lam, and K. Y. Sze, "Non-fragile output feedback $H_{\infty}$ vehicle suspension control using genetic algorithm," Engineering Applications of Artificial Intelligence, vol. 16, no. 7-8, pp. 667680, 2003.

[30] H. Chen and K.-H. Guo, "Constrained $H_{\infty}$ control of active suspensions: an LMI approach," IEEE Transactions on Control Systems Technology, vol. 13, no. 3, pp. 412-421, 2005.

[31] P. K. S. Tam and W. Tan, "A direct composite $H_{\infty}$ controller design for a two-time-scale active suspension system," in Proceedings of the 22nd IEEE International Conference on Industrial Electronics, Control, and Instrumentation (IECON '96), vol. 3, pp. 1401-1405, Taipei, Taiwan, August 1996.

[32] I. J. Fialho and G. J. Balas, "Design of nonlinear controllers for active vehicle suspensions using parameter-varying control synthesis," Vehicle System Dynamics, vol. 33, no. 5, pp. 351-370, 2000.

[33] J. K. Hedrick and T. Butsuen, "Invariant properties of automotive suspensions," Proceedings of the Institution of Mechanical Engineers, Part D: Journal of Automobile Engineering, vol. 204, no. 1, pp. 21-27, 1990.

[34] V. Blondel and J. N. Tsitsiklis, "NP-hardness of some linear control design problems," SIAM Journal on Control and Optimization, vol. 35, no. 6, pp. 2118-2127, 1997.

[35] V. L. Syrmos, C. T. Abdallah, P. Dorato, and K. Grigoriadis, "Static output feedback-a survey," Automatica, vol. 33, no. 2, pp. 125-137, 1997.

[36] H. Gao, Y. Zhao, and W. Sun, "Input-delayed control of uncertain seat suspension systems with human-body model," IEEE Transactions on Control Systems Technology, vol. 18, no. 3, pp. 591-601, 2010. 
[37] H. Du, W. Li, and N. Zhang, "Integrated seat and suspension control for a quarter car with driver model," IEEE Transactions on Vehicular Technology, vol. 61, no. 9, pp. 3893-3908, 2012.

[38] H. Zhang, Y. Shi, and A. S. Mehr, "Robust weighted $H_{\infty}$ filtering for networked systems with intermittent measurements of multiple sensors," International Journal of Adaptive Control and Signal Processing, vol. 25, no. 4, pp. 313-330, 2011.

[39] L. C. Félix-Herrán, D. Mehdi, J. de J. Rodríguez-Ortiz, R. Soto, and R. Ramírez-Mendoza, " $H_{\infty}$ control of a suspension with a magnetorheological damper," International Journal of Control, vol. 85, no. 8, pp. 1026-1038, 2012.

[40] H. Zhang, Y. Shi, and A. S. Mehr, "Robust $H_{\infty}$ PID control for multivariable networked control systems with disturbance/noise attenuation," International Journal of Robust and Nonlinear Control, vol. 22, no. 2, pp. 183-204, 2012.

[41] J. Liu, Y. Hu, and Z. Lin, "State-feedback $H_{\infty}$ control for LPV system using T-S fuzzy linearization approach," Mathematical Problems in Engineering, vol. 2013, Article ID 169454, 18 pages, 2013.

[42] H. Zhang, Y. Shi, and M. Liu, " $H_{\infty}$ step tracking control for networked discrete-time nonlinear systems with integral and predictive actions," IEEE Transactions on Industrial Informatics, vol. 9, no. 1, pp. 337-345, 2013.

[43] H. Zhang, J. Wang, and Y. Shi, "Robust $H_{\infty}$ sliding-mode control for markovian jump systems subject to intermittent observations and partially known transition probabilities," Systems and Control Letters, vol. 62, no. 12, pp. 1114-1124, 2013.

[44] S. Hu, D. Yue, Z. Du, and J. Liu, "Reliable $H_{\infty}$ non-uniform sampling tracking control for continuous-time non-linear systems with stochastic actuator faults," IET Control Theory \& Applications, vol. 6, no. 1, pp. 120-129, 2012.

[45] Y. Cui, K. Liu, Y. Zhao, and X. Wang, "Robust $H_{\infty}$ control for a class of uncertain switched fuzzy time-delay systems based on T-S models," Mathematical Problems in Engineering, vol. 2013, Article ID 234612, 8 pages, 2013.

[46] X. Li, H. R. Karimi, and Z. Xiang, "Robust reliable control of uncertain discrete impulsive switched systems with state delays," Mathematical Problems in Engineering, vol. 2013, Article ID 197819, 8 pages, 2013.

[47] L.-X. Guo and L.-P. Zhang, "Robust $H_{\infty}$ control of active vehicle suspension under non-stationary running," Journal of Sound and Vibration, vol. 331, no. 26, pp. 5824-5837, 2012. 


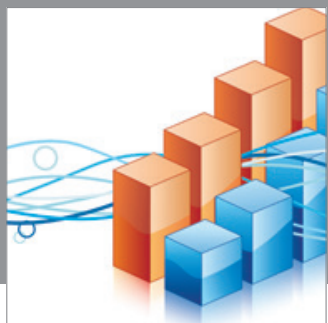

Advances in

Operations Research

mansans

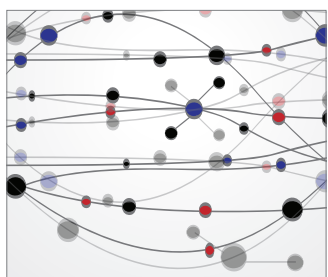

The Scientific World Journal
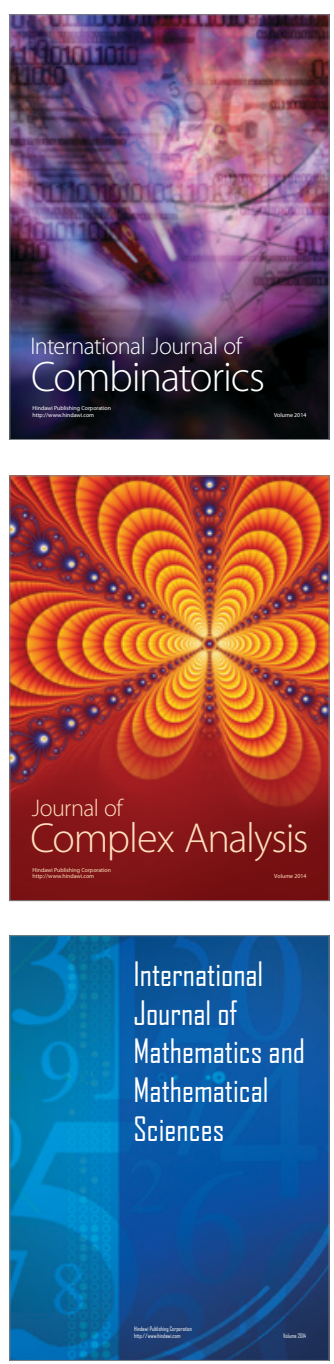
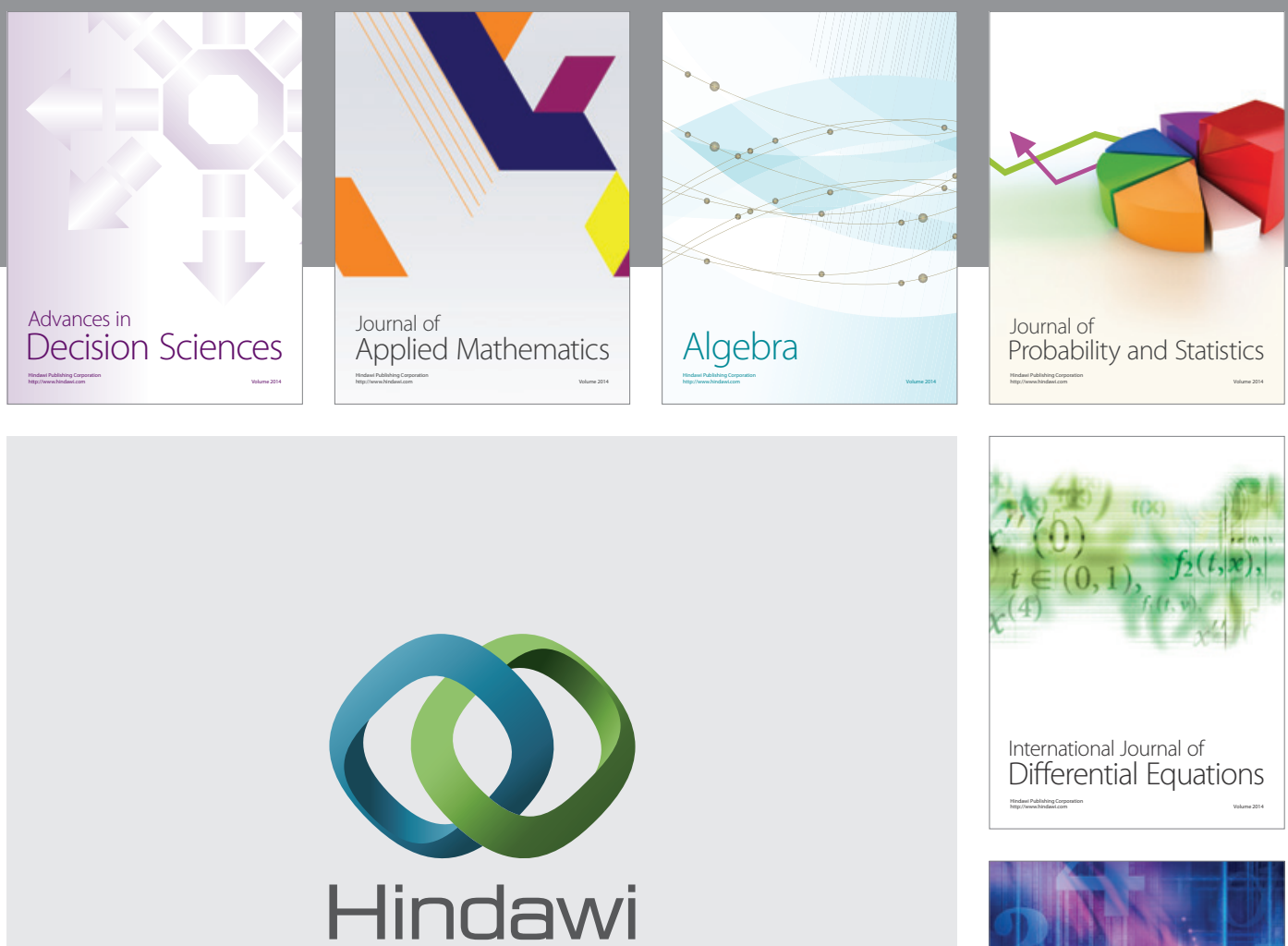

Submit your manuscripts at http://www.hindawi.com
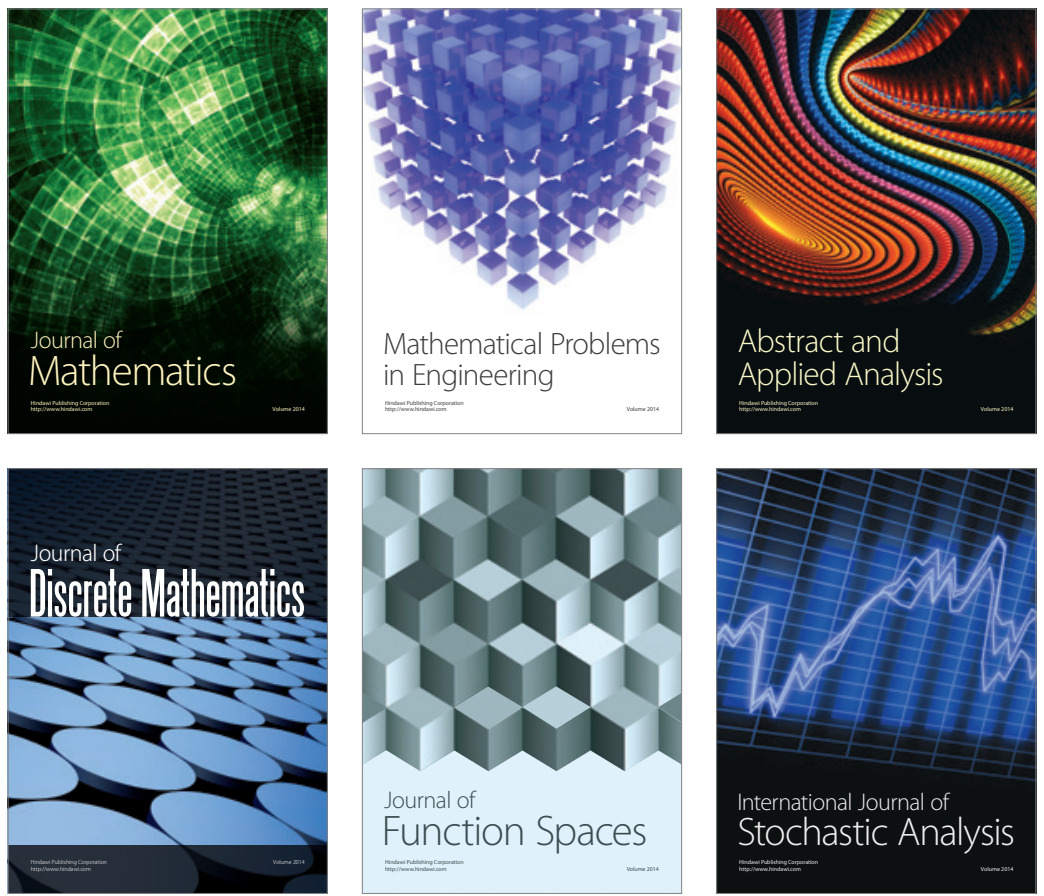

Journal of

Function Spaces

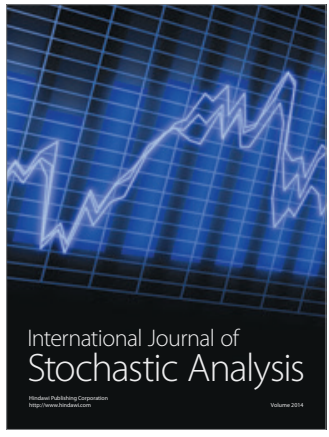

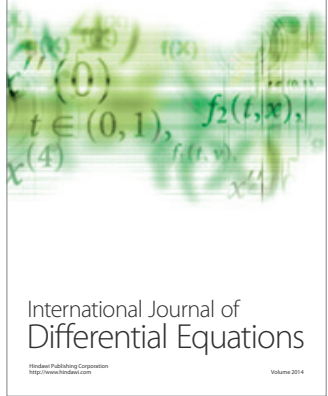
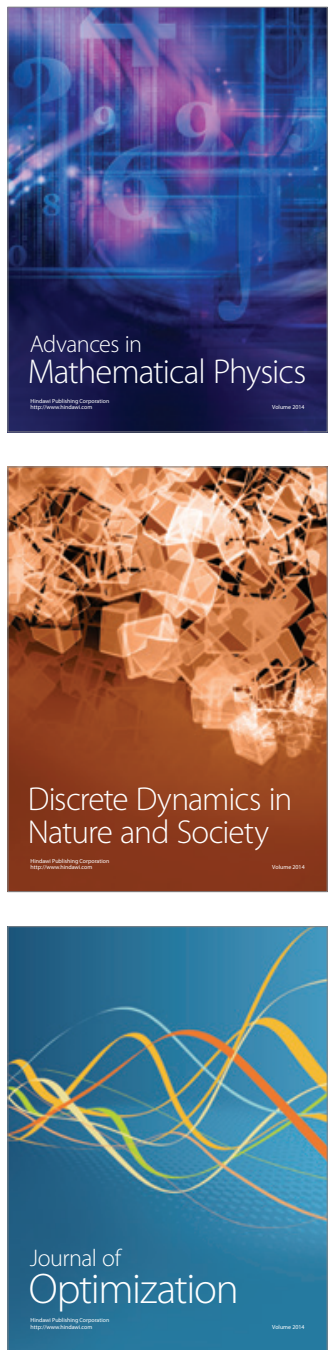